\title{
Numerical Analysis on Iron Loss of Reactor Core under Hysteresis $B-H$ Curve Using Play Model for DC-DC Converter
}

\author{
Shuangshuang Zhong ${ }^{* a)} \quad$ Non-member, Shunya Odawara* ${ }^{*}$ Member \\ Nguyen Gia Minh Thao* Non-member, Keisuke Fujisaki* Senior Member \\ Fujiyuki Iwamoto $^{* *} \quad$ Member, Tomonori Kimura** ${ }^{* *}$ Member \\ Takahiro Yamada* ${ }^{* *}$ Member
}

(Manuscript received Feb. 19, 2019, revised Oct. 28, 2019)

\begin{abstract}
The decrease in iron loss in a DC-DC converter is one of the most important issues for energy apparatuses. To reduce the iron loss under the magnetic hysteresis $B-H$ curve caused by the current ripples in the reactor core built in the DC-DC converter, where $B$ and $H$ are the magnetic flux density and magnetic field intensity, respectively, we apply a numerical calculation method based on a suitable magnetic hysteresis model using the play model. When the operating current (corresponding to the magnetic field), on the reactor core is constant, there are two points of magnetic flux density in the $B-H$ curve because of the nonlinear magnetic characteristics, the higher magnetic flux density and lower magnetic flux density. At the higher $B$ and lower $B$ operating points, minor loops appear in the $B-H$ curve when the operating current on the reactor core is varied and has ripples. The calculation results for all three cases show that the performance of the higher $B$ operating point is significantly better than that of the lower $B$ operating point. In particular, a higher $B$ operating point has lower iron loss, smaller magnetic flux density ripples, and larger energy density of the magnetic field caused by the smaller minor loop.
\end{abstract}

Keywords: DC-DC converter, play model, iron loss, minor loop, magnetic flux density ripples, current ripples

\section{Introduction}

In DC-DC converters, a reactor core is often used to store the magnetic energy. However, due to the ON and OFF modes of switching operation of the power semiconductor utilized in DC-DC converters, the operating current on the reactor core has ripples; as a result, minor loops are occurred in a magnetic hysteresis $B-H$ curve on the reactor core which substantially affect the iron loss of the reactor core. In order to improve overall efficiency of the DC-DC converter and reduce negative impacts on the environment, the iron loss of the reactor core used in the converter should be considered and decreased.

The authors in (1) reported the relationship between the DC-AC inverter circuit and the iron loss, where the $\mathrm{ON}$ and OFF modes in an inverter are clarified to be related to a magnetic hysteresis $B-H$ curve. Furthermore, the minor loops can be appeared in the $B-H$ curve due to the carrier frequency for switching operation of the power semiconductors and the voltage drop of the power semiconductors ${ }^{(2)-(5)}$. In addition, the minor loops lead to increase or decrease the iron loss if they are the closed or open minor loops, respectively.

a) Correspondence to: Shuangshuang Zhong. E-mail: 848924359 @qq.com

* Toyota Technological Institute

2-12-1, Hisakata, Tenpaku-ku, Nagoya, Aichi 468-8511, Japan

${ }^{* *}$ DENSO CORPORATION

500-1, Minamiyama, Komenoki-cho, Nisshin, Aichi 470-0111, Japan
In a DC-DC converter, the minor loops can be occurred in the $B-H$ curve when the operating current on the reactor core is changed and has ripples because the ON or OFF time of switching operation of the power semiconductor is changed. The change of the ON or OFF time of switching operation can produce the up-mode or down-mode current waveform, which has different and crucial influences on the minor loops in the $B-H$ curve. Thus, the influence of changing the $\mathrm{ON}$ or OFF time of switching operation of the power semiconductor used in DC-DC converter on the minor loops in the $B-H$ curves should be considered; in fact, this issue has not yet been investigated in existing studies.

To evaluate the iron loss and investigate a method for efficiently reducing it, we apply a numerical analysis method based on the play model ${ }^{(6)-(8)}$, which requires smaller computational cost than the Preisach model because of its simple mathematical description and has an equivalent capability compared to the Preisach model ${ }^{(6)}$ in depicting the magnetic hysteresis properties.

It is noted that this paper mainly focuses on the numerical analysis on iron loss of the reactor core under the hysteresis $B-H$ curve using a play model for DC-DC converters; experimental investigations to compare with the numerical simulation results, which are useful but beyond the main scope of this paper, will be intensively researched in our next work. The remaining of this paper is organized as follows. Section 2 briefly presents the magnetic property of the reactor core utilized in the DC-DC converter. Section 3 shows the numerical calculation model and calculation method designed 
particularly for the study. Section 4 gives analysis results in the first case where the magnitude range and fundamental frequency of the smaller current ripples are $\Delta i=0.1 \mathrm{~A}$ and $f_{\mathrm{o}}=$ $50 \mathrm{~Hz}$, respectively; this section also presents a comprehensive analysis of classical eddy current loss, hysteresis loss, magnetic flux density ripples and energy density of magnetic field. Furthermore, two additional cases in simulation, where the larger magnitude range and higher fundamental frequency of the smaller current ripples are input to the reactor core, are considered in Section 5 to validate effectiveness of the proposed method. The conclusion and future work are described in the last section.

\section{Magnetic Property in DC-DC Converter}

As depicted in Fig. 1, an insulated gate bipolar transistor, i.e. power semiconductor $\mathrm{Q}$, is used as the switching device; the DC-DC boost converter has two operation modes, namely ON mode and OFF mode. In this research, the two modes switch at intersection points with the fundamental frequency of $f_{\mathrm{c}}=50 \mathrm{~Hz}$ in the first two simulation cases and $f_{\mathrm{o}}=100 \mathrm{~Hz}$ in the last case.

In the ON mode as shown in Fig. 1(a), there are two loops of current in the circuit. As marked in the bold lines, the first current $i_{\mathrm{L}}$ flows from the DC voltage source, through the reactor core, switching device $\mathrm{Q}$, and back to the $\mathrm{DC}$ voltage source; the other $i_{\mathrm{C}}$ flows through the capacitance, resistance, and back to the capacitance. Therefore, the voltage $V_{\mathrm{L}}$ on the reactor core is,

$$
V_{\mathrm{L}}=E \text {. }
$$

where $E$ is the voltage of the DC voltage source.

In the OFF mode as illustrated in Fig. 1(b), the current flows from the DC voltage source, through the reactor core, diode, resistance or capacitance and back to the DC voltage source as marked in the bold lines. Thus, the $V_{\mathrm{L}}$ now is

$$
V_{\mathrm{L}}=E-V_{\mathrm{C}}=E-V_{\mathrm{R}}
$$

where $V_{\mathrm{C}}$ is the capacitance voltage and $V_{\mathrm{R}}$ is the load voltage; due to the parallel circuit, $V_{\mathrm{C}}=V_{\mathrm{R}}$.

As a result, the voltage $V_{\mathrm{L}}$ on the reactor core used in the DC-DC converter is described in Fig. 2(a), where $T_{1}, T_{2}$ and $T=1 / f_{\mathrm{c}}=0.02 \mathrm{~s}$ are the OFF time, ON time and one period of switching operation of the power semiconductor built in the DC-DC converter, respectively. Due to the change of voltage on the reactor core, the operating current $i_{\mathrm{L}}$ on the reactor core has ripples $\Delta i$ as depicted in Fig. 2(b). Because the operating current $i_{\mathrm{L}}$ has ripples, the minor loops can be happened in the $B-H$ curve around the magnetic flux density operating points corresponding to the operating current, as shown in Fig. 2(c).

In the $B-H$ curve, there are two different points of the magnetic flux density with the same magnetic field intensity corresponding to the same operating current $i_{0}$ because of the nonlinear magnetic characteristics; in detail, the first one is the higher magnetic flux density $B_{\mathrm{a}}$ and the other is the lower magnetic flux density $B_{\mathrm{b}}$ as represented in Fig. 2(c). In addition, since the operating current $i_{\mathrm{L}}$ on the reactor core has ripples, the minor loops can be appeared at the operating points with the higher magnetic flux density $B_{\mathrm{a}}$ and the lower magnetic flux density $B_{\mathrm{b}}$ in the $B-H$ curve.

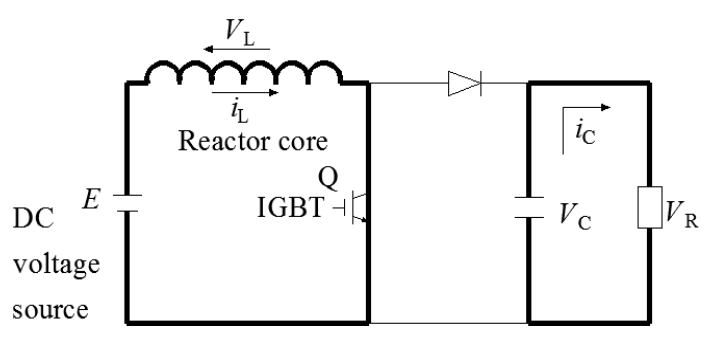

(a) ON mode

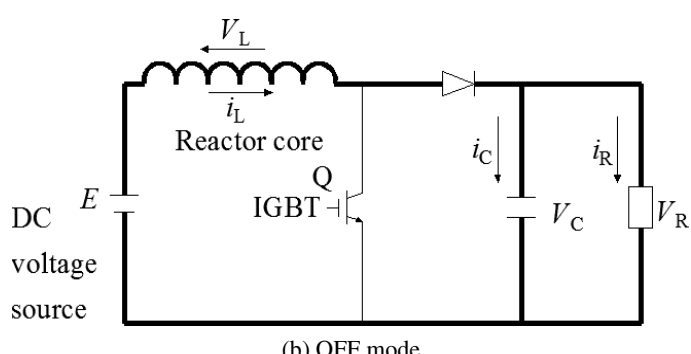

(b) OFF mode

Fig. 1. Current flows in DC-DC converter

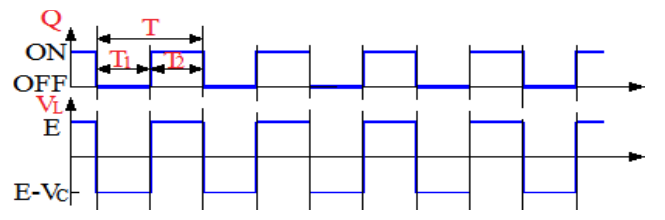

(a) Voltage waveform
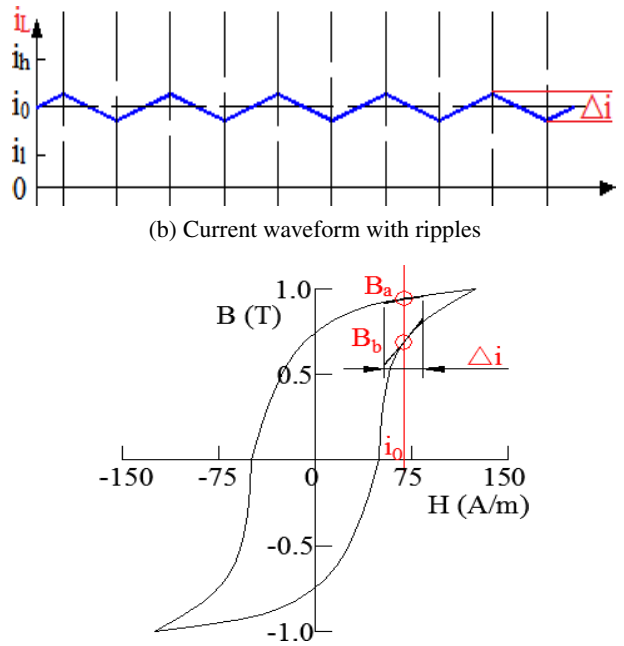

(c) $B$ - $H$ curve

Fig. 2. Magnetic property in DC-DC converter

Figure 3 gives the operating waveforms of the reactor core utilized in the DC-DC converter when we change the ON or OFF time of the switching device $\mathrm{Q}$ built in the converter to adjust the operating current $i_{\mathrm{L}}$ on the reactor core. As illustrated in Fig. 3(a), when we change the OFF time of the switching device $\mathrm{Q}$ in the third period; this means that the OFF time of the switching device $\mathrm{Q}$ changes from $T_{1}$ to $T_{3}$, and the down-mode current waveform can be obtained. As a result, the operating current changes from the point a, and its average amplitude value now varies from $i_{\mathrm{h}}$ to $i_{0}$; this will cause a minor loop to be appeared at the operating point of the higher magnetic flux density $B_{\mathrm{a}}$. Furthermore, we can also change the ON time of the switching device $\mathrm{Q}$ in the third period to get the up-mode current waveform as depicted 


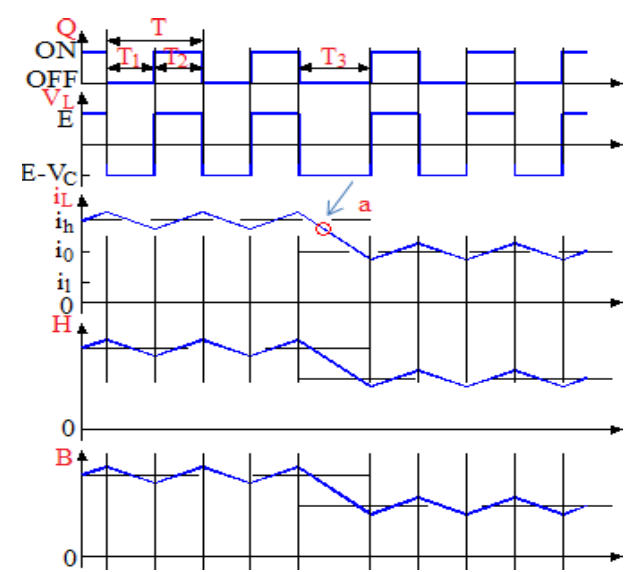

(a) Down-mode current waveform (changing OFF time of switching device Q)

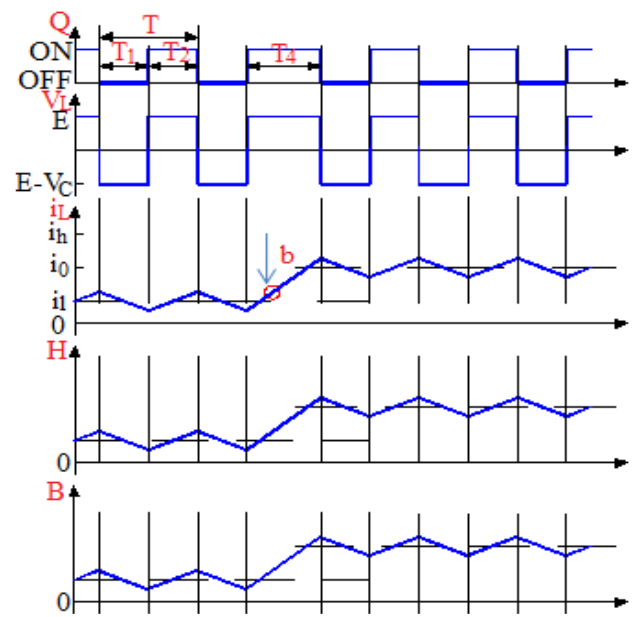

(b) Up-mode current waveform (changing ON time of switching device Q)

Fig. 3. Operating waveforms in DC-DC converter

in Fig. 3(b); this means that the ON time of the switching device $\mathrm{Q}$ changes from $T_{2}$ to $T_{4}$. The operating current changes from the point $\mathrm{b}$, and its average amplitude value changes from $i_{1}$ to $i_{0}$. As a consequence, a minor loop can be occurred at the operating point of the lower magnetic flux density $B_{\mathrm{b}}$.

The iron losses in the two current conditions may be different. In order to analyze and compare the sizes of the minor loops occurred in the two different conditions, we apply a numerical analysis method based on a magnetic hysteresis model using the play model as will be presented in the next section.

\section{Numerical Calculation Model and Calculation Method}

3.1 Play Model In order to consider the magnetic hysteresis properties on the reactor core built in the DC-DC converter which is given in Fig. 1, the magnetic hysteresis analysis with play model ${ }^{(6)(7)}$ is carried out.

Generally, in a magnetic analysis, the magnetic flux density is calculated with the magnetic field intensity as an input. However, with the play model, the magnetic field intensity can be obtained where the magnetic flux density is used as an input (9).

Figure 4(a) illustrates the property of play hysteron, which is expressed as

$$
p_{\xi}(B)=\max \left(\min \left(p_{\xi}^{0}, B+\xi\right), B-\xi\right)
$$

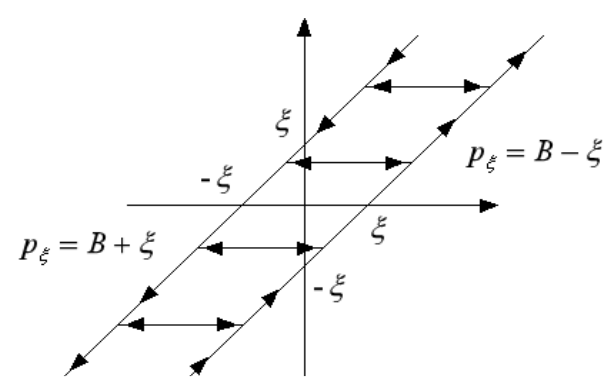

(a) Play hysteron

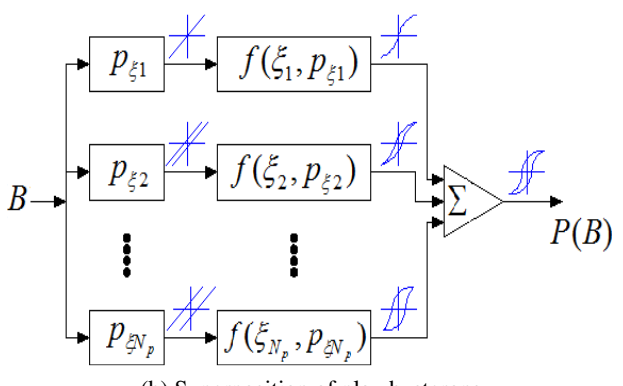

(b) Superposition of play hysterons

Fig. 4. Play model

where $\xi$ is a nonnegative parameter representing the width of play hysteron, and $p_{\xi}^{0}$ is the value of $p_{\xi}$ at the previous time point.

As shown in Fig. 4(b), the play model describes the hysteretic relation between the input $B$ and the output $H$ by summing up play hysterons various $\xi$, and $\xi=\xi_{1}, \xi_{2}, \ldots$, as follows,

$$
H=P(B)=\sum_{n=1}^{N_{p}} f\left(\xi_{n}, p_{\xi n}(B)\right)
$$

where $N_{\mathrm{p}}$ is the number of play hysterons; $p_{\xi n}$ which $n$ is equal to $1, \ldots, N_{\mathrm{p}}$ are play hysterons with the width of $\xi_{n}$; $f$ is a single-valued function of $\xi$ and $p_{\xi}$, and $f$ is called the shape function, as follows (10),

$$
f(\xi, p)=f(\xi,|p|) \frac{p}{|p|}
$$

The play model represents the dc hysteresis $H_{\mathrm{DC}}(B)$, namely direct current hysteresis, due to its rate-independent property. From the $H_{\mathrm{DC}}(B)$, the ac magnetic field $H_{\mathrm{AC}}(B)$, that is the alternating current magnetic field, can be decomposed by

$$
H_{\mathrm{A} C}(B)=H_{\mathrm{D} C}(B)+k \frac{\sigma d}{12} \frac{\mathrm{d} B}{\mathrm{~d} t} .
$$

where $k$ is the anomaly factor, $2 ; \sigma$ is the electric conductivity, $1.92 \times 10^{6} \mathrm{~s} / \mathrm{m} ; d$ is the thickness of an electrical steel sheet, $0.35 \mathrm{~mm}$. The direct magnetic properties $H_{\mathrm{DC}}(B)$ is obtained by a scalar play model; as described in Fig. 5, there are $28 B$ - $H$ curves obtained from $B_{\max }=0.05 \mathrm{~T}$ to $B_{\max }=1.4 \mathrm{~T}$, where each step of the magnetic flux density is $0.05 \mathrm{~T}$.

3.2 Calculation Model In numerical calculation of this research, electrical steel sheets used for the ring core are made of a non-oriented material, namely $35 \mathrm{H} 300$, with the thickness of $0.35 \mathrm{~mm}$. The schematic diagram and specifications of the ring core are depicted in Fig. 6 and Table 1, respectively. 
Figure 7 illustrates the cross-sectional view of the ring core in Fig. 6 from the viewpoint of yz plane. In addition, a partial enlarged view of Fig. 7 which model is used in simulation is described in Fig. 8(a). In the process of numerical analysis, the simulation model is developed in JMAG software. Because the electrical steel sheets are not very tightly laminated in actual samples, the air gap between the electrical steel sheets is added in the process of the designed 3D model with JMAG. The 3D simulation model and its related dimensions are presented in Fig. 8(a). Furthermore, the simulation model is meshed and the partial enlarged view of Fig. 8(a) is shown in Fig. 8(b), which is the finite element model; the number of elements and nodes are 17524 and 35828, respectively.

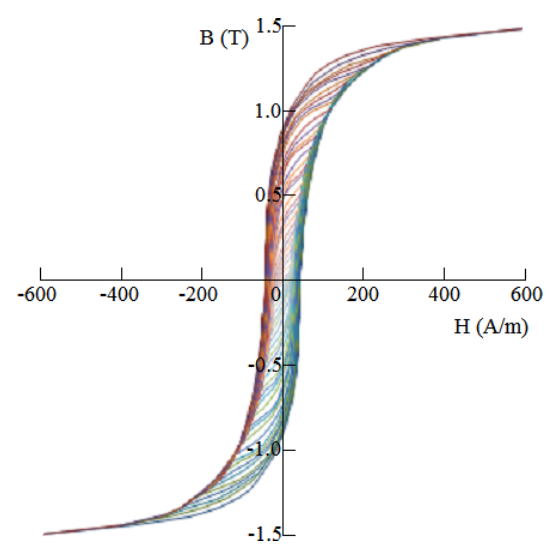

Fig. 5. $B-H$ curves for calculating $H_{\mathrm{DC}}(B)$ obtained from sinusoidal excitation by measurement

In JMAG, the copper coils on the ring core are excited by a current source; in the left coils, the current flows along the positive direction of $\mathrm{z}$ axis; in the right coils, the current flows along the negative direction of $\mathrm{z}$ axis, as described in Fig. 8(a). According to Ampere's rule, the direction of the magnetic flux density generated by the current is along the negative direction of $x$ axis. Therefore, the boundary conditions of plane $\mathrm{c}$ and plane $\mathrm{d}$ on the $\mathrm{xy}$ plane are set to be symmetric boundary; the boundary condition of plane a on the yz plane is set to be translation periodic boundary, and the periodic distance is $72 \mu \mathrm{m}$; whereas, the boundary conditions of plane e and plane $f$ on the xz plane and plane $b$ on the yz plane are set to be free boundary. In order to reduce the variation of magnetic flux, the eddy current is produced and its direction is to hinder the change of magnetic flux. Also, due to the existence of eddy current, the eddy current loss is generated; as a result, the total iron loss of the ring core increases.

3.3 Exciting Current for Different Points In order to get different operating points of the magnetic flux density

Table 1. Specifications of ring core

\begin{tabular}{|c|c|}
\hline Material & $35 \mathrm{H} 300$ \\
\hline Outside diameter $\varphi_{0}$ & $127 \mathrm{~mm}$ \\
\hline Inside diameter $\varphi_{1}$ & $102 \mathrm{~mm}$ \\
\hline Height $h$ & $7 \mathrm{~mm}$ \\
\hline Coil winding number $N_{1}$ & $254 \mathrm{turns}$ \\
\hline Cross-sectional area $S$ & $87.5 \mathrm{~mm}^{2}$ \\
\hline Magnetic-path length $L$ & $360 \mathrm{~mm}$ \\
\hline
\end{tabular}

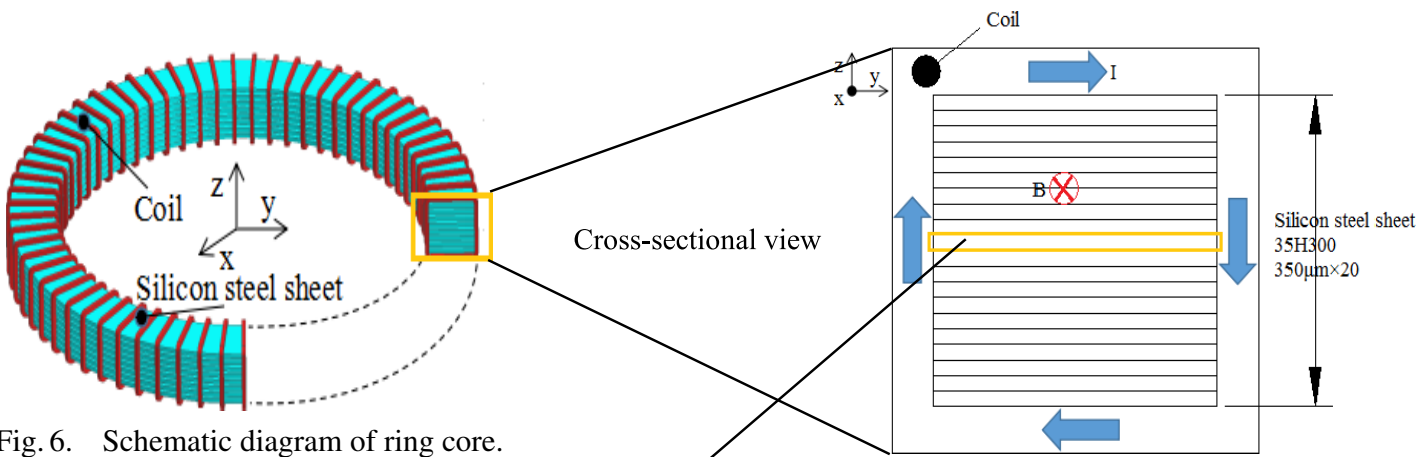

Fig. 6. Schematic diagram of ring core.

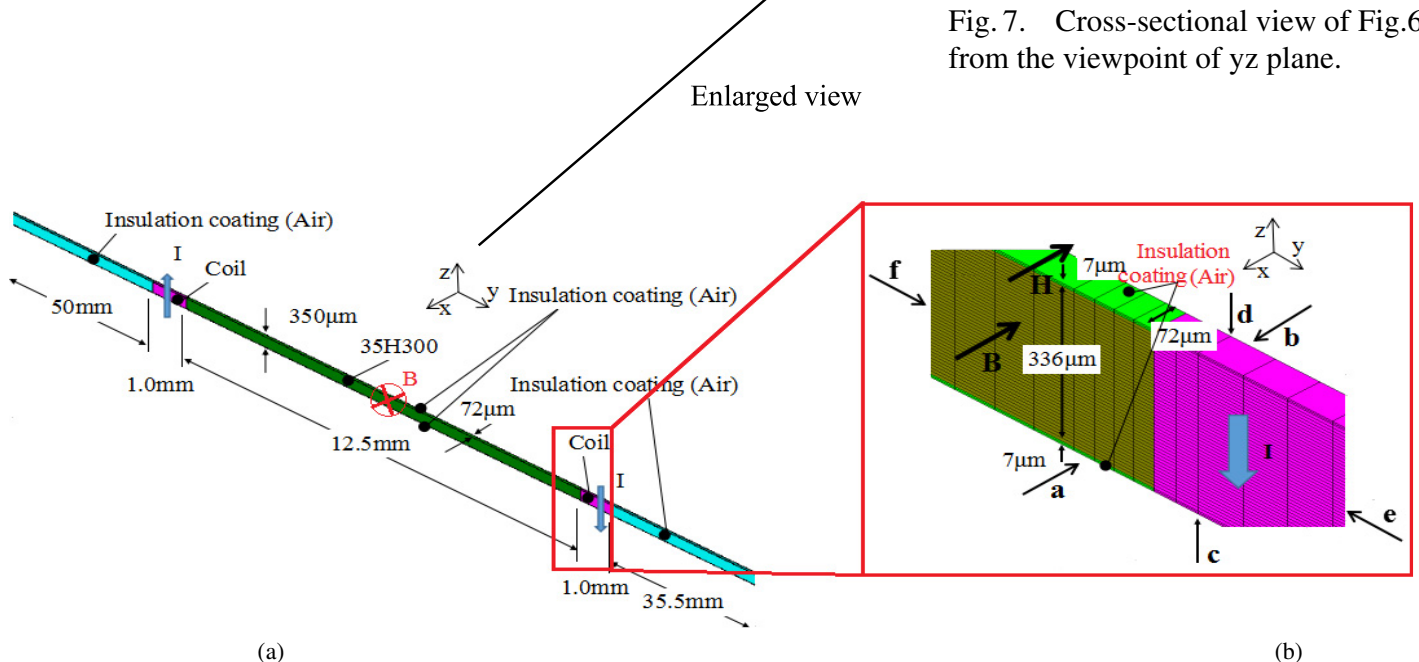

Fig. 8. 3D model; (a) partial enlarged view of Fig. 7, and (b) partial enlarged view of Fig. 8(a), i.e. 3D finite element model 
where minor loops can be happened in the same $B$ - $H$ curve with the same magnetic field intensity corresponding to the same operating current, we need to supply the exciting current for the ring core according to the following steps ${ }^{(11)}$.

First, due to the effects of ON and OFF modes in the DCDC converter, the operating current on the reactor core has ripples. In addition, because the sinusoidal waveform can be considered as fundamental characteristics for the triangular waveform of the current on the reactor core built in the DCDC converter, the input current with sinusoidal waveform is considered in this study as illustrated in Fig. 9.

After that, from the previous section, we can see that the operating current can be changed by adjusting the ON or OFF time of switching operation of the power semiconductor used in the DC-DC converter. Therefore, in the third operational period as depicted in Fig. 9, the average amplitude value of the operating current is increased from 0 to $i_{\mathrm{c} 0}$. Furthermore, when we reduce the OFF time of switching operation of the power semiconductor, the smaller current ripples start

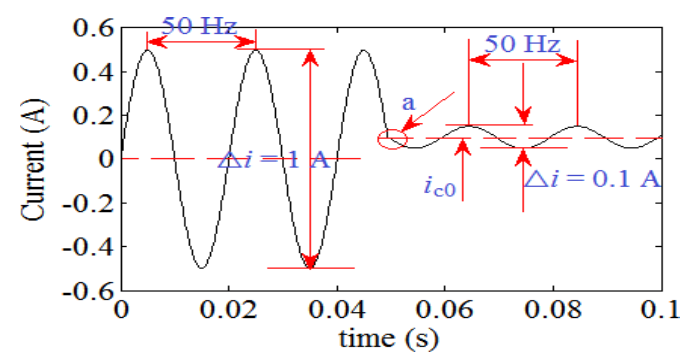

(a) Down-mode input current waveform (changing current at point a)

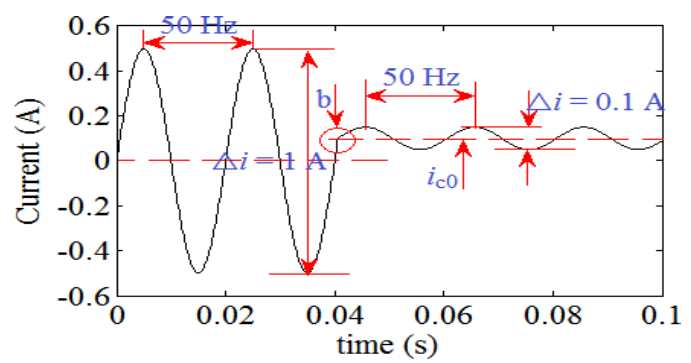

(b) Up-mode input current waveform (changing current at point b)

Fig. 9. Exciting current, Case 1 to flow from the point a at the end of the larger current ripple waveform and the down-mode current waveform is obtained as shown in Fig. 9(a). And when we reduce the ON time of switching operation of the power semiconductor, the smaller current ripples start to flow from the point $b$ at the end of the larger current ripple waveform and the up-mode current waveform is gained as described in Fig. 9(b). In this figure, the frequency of the larger or smaller current ripples is always $50 \mathrm{~Hz}$.

\section{Analysis Results in Case 1: The Smaller Cur- rent Ripples with Magnitude Range of $0.1 \mathrm{~A}$ and Fundamental Frequency of $50 \mathrm{~Hz}$}

Firstly, with the magnetic properties of the electrical steel sheet $35 \mathrm{H} 300$ used for the ring core, the magnetic hysteresis $B-H$ curve of the ring core is illustrated in Fig. 10(a-1) and Fig. 10(b-1).

After that, because the average amplitude value of the operating current is increased as shown in Fig. 9 and the new operating current $i_{\mathrm{c} 0}$ has ripples, the minor loops are appeared in the $B-H$ curves as given in Fig. 10(a-1) and Fig. 10(b-1). In addition, with the same magnetic field intensity corresponding to the same operating current $i_{\mathrm{c} 0}$, there are two different points of the magnetic flux density in the same $B$ - $H$ curve because of the nonlinear magnetic characteristics; in detail, the first one is the higher magnetic flux density $B_{\mathrm{a}}$, and the other is the lower magnetic flux density $B_{\mathrm{b}}$.

When the small current ripples start to flow at the point a at the end of the large current ripple waveform, namely the input current is with the down-mode current waveform, the minor loop is happened at the operating point of the magnetic flux density $B_{\mathrm{a}}$ in the $B-H$ curve. And when the small current ripples start to flow at the point $b$ at the end of the large current ripple waveform, namely the input current is now with the up-mode current waveform, the minor loop is occurred at the operating point of the magnetic flux density $B_{\mathrm{b}}$ in the $B-H$ curve. Enlargements of the minor loops appeared in the $B-H$ curves are presented in Fig. 10(a-2) and Fig. 10(b-2). From the figure, it can be seen that the two different operating points of the magnetic flux density, i.e. $B_{\text {a }}$ and $B_{\mathrm{b}}$, have different sizes of the minor loops. This means
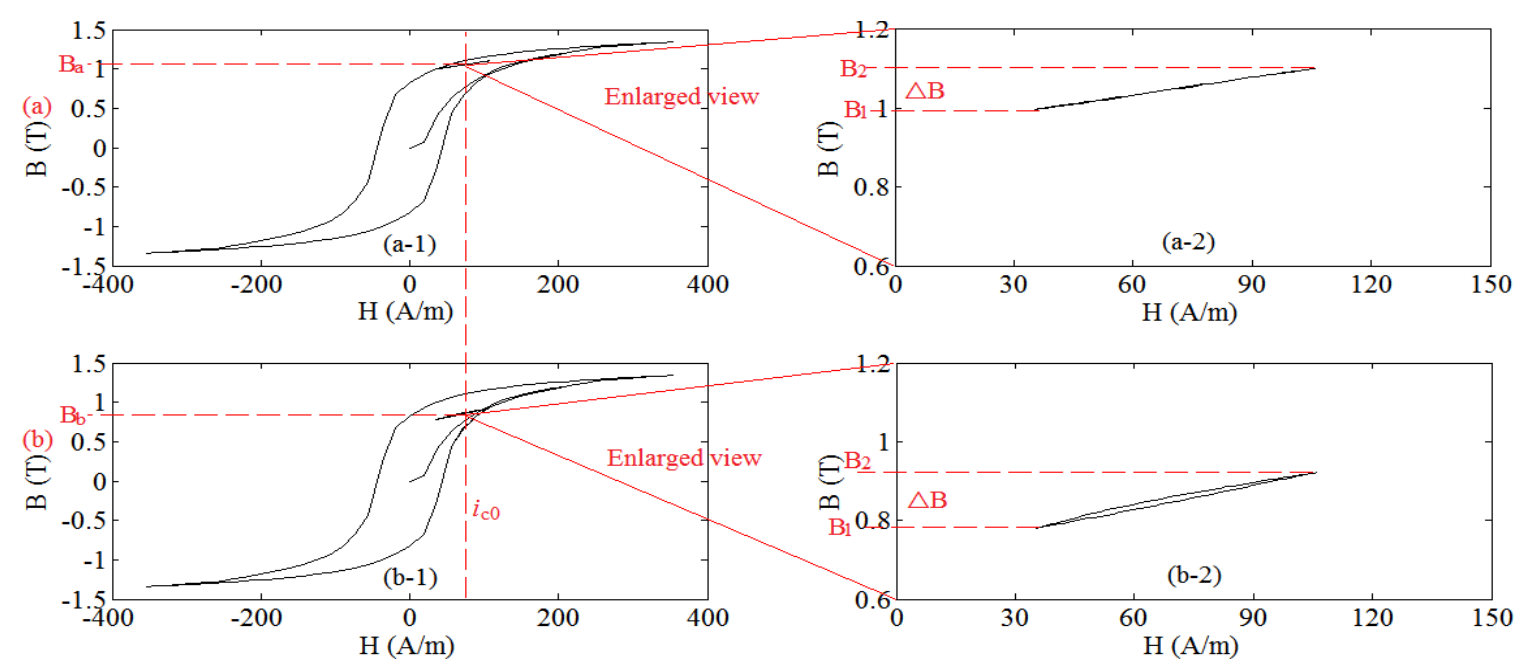

Fig. 10. Comparison on $B-H$ curves and enlargement of minor loops, Case 1; (a) down mode, namely higher magnetic flux density, and (b) up mode, namely lower magnetic flux density 
Table 2. Iron losses and magnetic properties between the lower operating point $B_{\mathrm{b}}$ and the higher operating point $B_{\mathrm{a}}$, Case 1

\begin{tabular}{|c|c|c|}
\hline & $\begin{array}{c}\text { Up mode, } \\
\text { operating point } B_{\mathrm{b}}\end{array}$ & $\begin{array}{c}\text { Down mode, } \\
\text { operating point } B_{\mathrm{a}}\end{array}$ \\
\hline Iron loss $W_{\mathrm{Fe}}(\mathrm{W} / \mathrm{kg})$ & $4.89 \times 10^{-3}$ & $0.576 \times 10^{-3}$ \\
\hline $\begin{array}{c}\text { Classical eddy current loss } \\
W_{\text {eddy,classic }}(\mathrm{W} / \mathrm{kg})\end{array}$ & $0.577 \times 10^{-3}$ & $0.314 \times 10^{-3}$ \\
\hline Hysteresis loss $W_{\text {hys }}(\mathrm{W} / \mathrm{kg})$ & $4.313 \times 10^{-3}$ & $0.262 \times 10^{-3}$ \\
\hline Magnetic flux density $B(\mathrm{~T})$ & 0.851 & 1.05 \\
\hline $\begin{array}{c}\text { Magnetic flux density } \\
\text { ripples } \Delta B(\mathrm{~T})\end{array}$ & 0.143 & 0.106 \\
\hline $\begin{array}{c}\text { Energy density of magnetic } \\
\text { field } \varepsilon\left(\mathrm{W} / \mathrm{m}^{3}\right)\end{array}$ & 30.021 & 37.041 \\
\hline
\end{tabular}

that the iron losses resulted from the minor loops of ring core obtained with the two different kinds of input current waveforms illustrated in Fig. 9 are significantly different.

The iron losses caused by the minor loops of ring core obtained with the down-mode and the up-mode input current waveforms are presented and compared in Table 2. The iron loss $W_{\mathrm{Fe}}$ can be calculated by Eq. (7), and the classical eddy current loss $W_{\text {eddy,classic }}$ is computed by Eq. (8) ${ }^{(12)}$. Because the abnormal eddy current loss is ignored in the research, the hysteresis loss $W_{\text {hys }}$ is reckoned by Eq. (9).

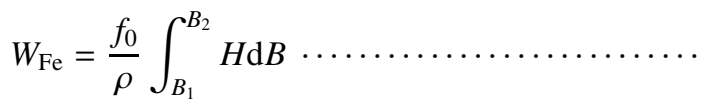

$$
\begin{aligned}
& W_{\text {eddy,classic }}=\frac{1}{T} \sum_{t=1}^{\text {step }} \frac{1}{V_{(\text {total }}} \sum_{i e=1}^{n} \frac{J_{(\mathrm{ie})}(t)^{2}}{\sigma_{(\mathrm{ie})}} V_{(\mathrm{ie})} \Delta t \ldots \\
& W_{\text {hys }}=W_{\mathrm{Fe}}-W_{\text {eddy,classic }} \\
& \varepsilon=\frac{1}{2} B H=\frac{1}{2} L I^{2}
\end{aligned}
$$

Here, $\rho$ is the density of the electrical steel sheets, $7650 \mathrm{~kg} / \mathrm{m}^{3} ; f_{0}$ is the fundamental frequency, $50 \mathrm{~Hz} ; V$ is the volume of the steel sheets, $\mathrm{m}^{3} ; n$ is the number of elements; (ie) means the element number; $J$ is the eddy current density, $\mathrm{A} / \mathrm{m}^{2} ; L$ is the inductance of the ring core, $\mathrm{H} ; I$ is the current on the reactor core, $\mathrm{A}$.

This table shows that the iron loss generated by the minor loop with the higher magnetic flux density operating point $B_{\text {a }}$ corresponding to the down-mode input current waveform is substantially smaller than the one with the lower magnetic flux density operating point $B_{\mathrm{b}}$ corresponding to the up-mode input current waveform resulting from the much smaller size of the minor loop appeared at the operating point $B_{\mathrm{a}}$ in the $B-H$ curve. In detail, the iron loss produced by the minor loop with the lower operating point $B_{\mathrm{b}}$ is $4.89 \times 10^{-3} \mathrm{~W} / \mathrm{kg}$, while the one with the higher operating point $B_{\mathrm{a}}$ is $0.576 \times 10^{-3} \mathrm{~W} / \mathrm{kg}$; the reduction in percentage is $88.22 \%$. The classical eddy current loss and hysteresis loss of the lower operating point $B_{\mathrm{b}}$ are $W_{\text {eddy,classic }}=$ $0.577 \times 10^{-3} \mathrm{~W} / \mathrm{kg}$ and $W_{\text {hys }}=4.313 \times 10^{-3} \mathrm{~W} / \mathrm{kg}$, respectively, whereas, the classical eddy current loss and hysteresis loss of the higher operating point $B_{\mathrm{a}}$ are $W_{\text {eddy,classic }}=$ $0.314 \times 10^{-3} \mathrm{~W} / \mathrm{kg}$ and $W_{\text {hys }}=0.262 \times 10^{-3} \mathrm{~W} / \mathrm{kg}$, respectively.

Table 2 also illustrates that the detailed values of the lower operating point $B_{\mathrm{b}}$ and the higher operating point $B_{\mathrm{a}}$ are
$0.851 \mathrm{~T}$ and $1.05 \mathrm{~T}$, respectively. Furthermore, the magnetic flux density ripples $\Delta B$ in the minor loops occurred at the lower operating point $B_{\mathrm{b}}$ and the higher operating point $B_{\mathrm{a}}$ are $0.143 \mathrm{~T}$ and $0.106 \mathrm{~T}$, respectively. In addition, the energy density of magnetic field $\varepsilon$ computed by Eq. (10) at the lower operating point $B_{\mathrm{b}}$ and the higher operating point $B_{\mathrm{a}}$ are $30.021 \mathrm{~W} / \mathrm{m}^{3}$ and $37.041 \mathrm{~W} / \mathrm{m}^{3}$, respectively. According to these results, the efficacy with the higher operating point $B_{\mathrm{a}}$ in reducing the iron loss and magnetic flux density ripples caused by the minor loops as well as improving the energy density of magnetic field resulted from the minor loops is remarkably better than the one with the lower operating point $B_{\mathrm{b}}$.

\section{Analysis Results in Two Additional Cases}

In this section, two additional simulation cases, where the larger magnitude range $\Delta i$ and the higher fundamental frequency $f_{\mathrm{o}}$ of the smaller current ripples are input to the reactor core as presented in Case 2 and Case 3, respectively, are conducted to carefully validate the efficacy of the proposed calculation method.

5.1 Case 2: The Smaller Current Ripples with Magnitude Range of $0.16 \mathrm{~A}$ and Fundamental Frequency of $50 \mathrm{~Hz}$ In this case, the magnitude range and fundamental frequency of the smaller current ripples are set to be $\Delta i$ $=0.16 \mathrm{~A}$ and $f_{\mathrm{o}}=50 \mathrm{~Hz}$, respectively, which the magnitude range of the smaller current ripples is increased to $160 \%$ as compared to the range $\Delta i=0.1 \mathrm{~A}$ in Case 1 , but the fundamental frequency of the smaller current ripples is the same with the one in Case 1, as depicted in Fig. 11. In addition, the magnitude range and fundamental frequency of the larger current ripples are not changed. As a result, the $B-H$ curves caused by the exciting current described in Fig. 11 and the enlarged view of the minor loops are shown in Fig. 12.

As illustrated in Fig. 12, the iron loss generated by the minor loop with the higher magnetic flux density operating point $B_{\mathrm{a}}$ corresponding to the down-mode input current waveform is also substantially smaller than the one with the

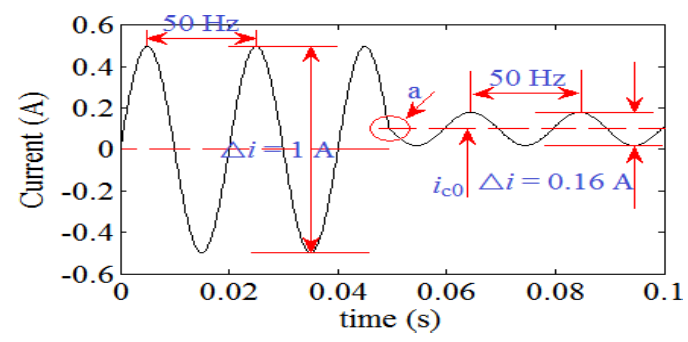

(a) Down-mode input current waveform (changing current at point a)

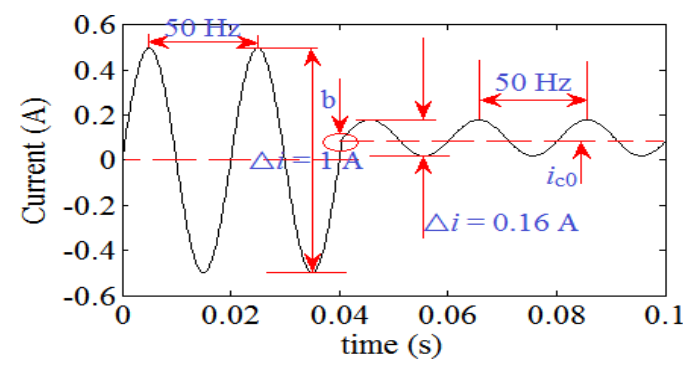

(b) Up-mode input current waveform (changing current at point b)

Fig. 11. Exciting current, Case 2 

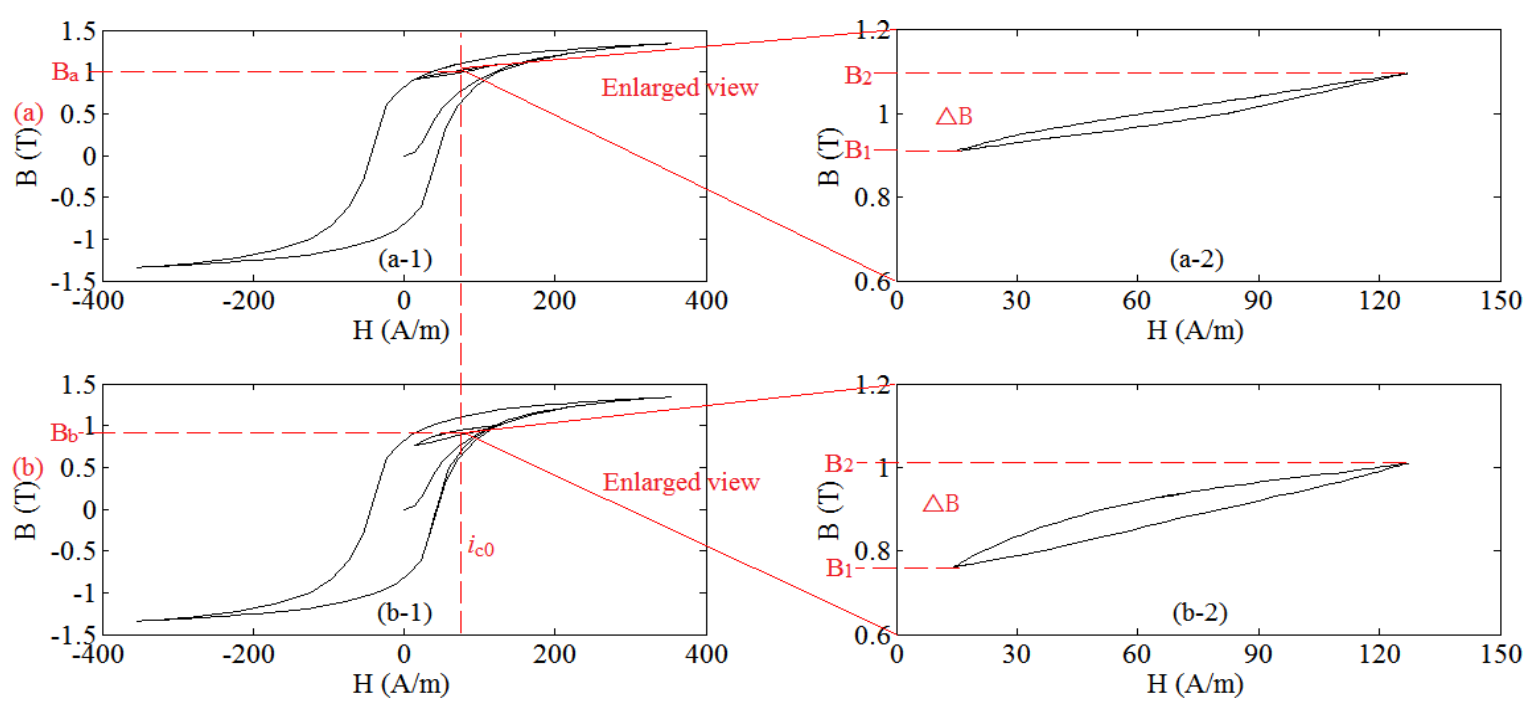

Fig. 12. Comparison on $B-H$ curves and enlargement of minor loops, Case 2; (a) down mode, namely higher magnetic flux density, and (b) up mode, namely lower magnetic flux density

Table 3. Iron losses and magnetic properties between the lower operating point $B_{\mathrm{b}}$ and the higher operating point $B_{\mathrm{a}}$, Case 2

\begin{tabular}{|c|c|c|}
\hline & $\begin{array}{c}\text { Up mode, } \\
\text { operating point } B_{\mathrm{b}}\end{array}$ & $\begin{array}{c}\text { Down mode, } \\
\text { operating point } B_{\mathrm{a}}\end{array}$ \\
\hline Iron loss $W_{\mathrm{Fe}}(\mathrm{W} / \mathrm{kg})$ & $3.07 \times 10^{-2}$ & $1.47 \times 10^{-2}$ \\
\hline $\begin{array}{c}\text { Classical eddy current loss } \\
W_{\text {cddy,classic }}(\mathrm{W} / \mathrm{kg})\end{array}$ & $0.418 \times 10^{-3}$ & $0.241 \times 10^{-3}$ \\
\hline Hysteresis loss $W_{\text {hys }}(\mathrm{W} / \mathrm{kg})$ & $3.0282 \times 10^{-2}$ & $1.4459 \times 10^{-2}$ \\
\hline Magnetic flux density $B(\mathrm{~T})$ & 0.897 & 1.01 \\
\hline $\begin{array}{c}\text { Magnetic flux density } \\
\text { ripples } \Delta B(\mathrm{~T})\end{array}$ & 0.249 & 0.186 \\
\hline $\begin{array}{c}\text { Energy density of magnetic } \\
\text { field } \varepsilon\left(\mathrm{W} / \mathrm{m}^{3}\right)\end{array}$ & 33.576 & 37.984 \\
\hline
\end{tabular}

lower magnetic flux density operating point $B_{\mathrm{b}}$ corresponding to the up-mode input current waveform resulting from the much smaller size of the minor loop appeared at the operating point of the higher $B$ in the $B-H$ curve. In detail, the iron losses produced by the minor loops at the operating point $B_{\mathrm{a}}$ and $B_{\mathrm{b}}$ are $1.47 \times 10^{-2} \mathrm{~W} / \mathrm{kg}$ and $3.07 \times 10^{-2} \mathrm{~W} / \mathrm{kg}$, respectively, as expressed in Table 3 . As compared to the operating point $B_{\mathrm{b}}$, the iron loss caused by the minor loop significantly decreases about $52.12 \%$ at the operating point $B_{\mathrm{a}}$. Moreover, the magnetic flux density ripples $B$ in the minor loops occurred at the lower operating point $B_{\mathrm{b}}$ and the higher operating point $B_{\mathrm{a}}$ are $0.249 \mathrm{~T}$ and $0.186 \mathrm{~T}$, respectively. The results of Case 2 in Table 3 have a same trend with the ones of Case 1 in Table 2.

5.2 Case 3: The Smaller Current Ripples with Magnitude Range of $0.1 \mathrm{~A}$ and Fundamental Frequency of $100 \mathrm{~Hz}$ In this case, the magnitude range of the smaller current ripples is set to be $\Delta i=0.1 \mathrm{~A}$ as in Case 1 , but the fundamental frequency of the smaller current ripples is now increased from $f_{\mathrm{o}}=50 \mathrm{~Hz}$ to be $f_{\mathrm{o}}=100 \mathrm{~Hz}$ as depicted in Fig. 13. The magnitude range and fundamental frequency of the larger current ripples are still not changed as compared to Case 1. After simulation, the $B-H$ curves caused by the exciting current shown in Fig. 13 and the enlarged view of the

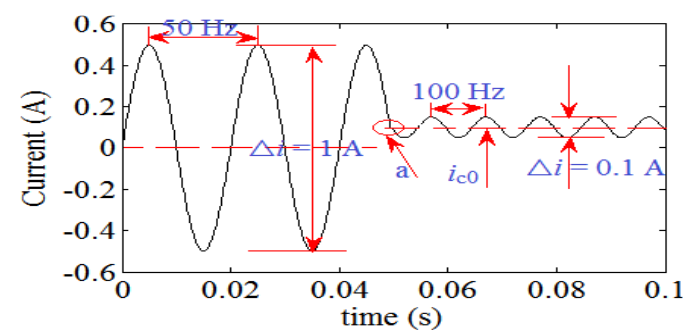

(a) Down-mode input current waveform (changing current at point a)

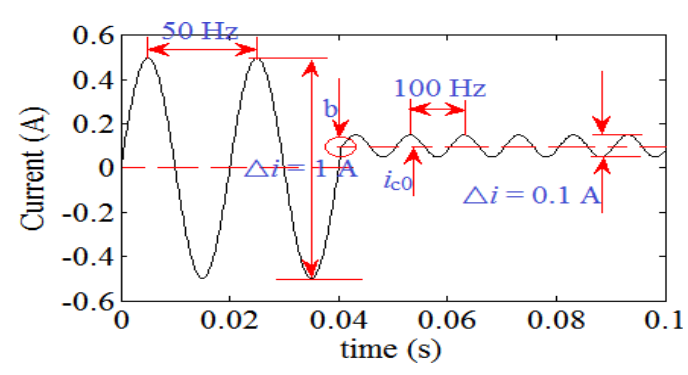

(b) Up-mode input current waveform (changing current at point b)

Fig. 13. Exciting current, Case 3

minor loops are obtained as presented in Fig. 14.

As depicted in Fig. 14, the iron loss generated by the minor loop with the higher magnetic flux density operating point $B_{\text {a }}$ corresponding to the down-mode input current waveform is still substantially smaller than the one with the lower magnetic flux density operating point $B_{\mathrm{b}}$ corresponding to the up-mode input current waveform resulting from the much smaller size of the minor loop appeared at the operating point of the higher $B$ in the $B-H$ curve. In detail, the iron losses produced by the minor loops at the operating points $B_{\text {a }}$ and $B_{\mathrm{b}}$ are $0.533 \times 10^{-3} \mathrm{~W} / \mathrm{kg}$ and $3.1 \times 10^{-3} \mathrm{~W} / \mathrm{kg}$, respectively, as described in Table 4. As compared to the operating point $B_{\mathrm{b}}$, the iron loss caused by the minor loop notably reduces about $82.81 \%$ at the operating point $B_{\mathrm{a}}$. Besides, the magnetic flux density ripples $\Delta B$ in the minor loops occurred at the lower operating point $B_{\mathrm{b}}$ and the higher operating point $B_{\mathrm{a}}$ are $0.141 \mathrm{~T}$ and $0.104 \mathrm{~T}$, respectively. The results of Case 3 in Table 4 also have a same trend with the ones of Case 1 in Table 2. 

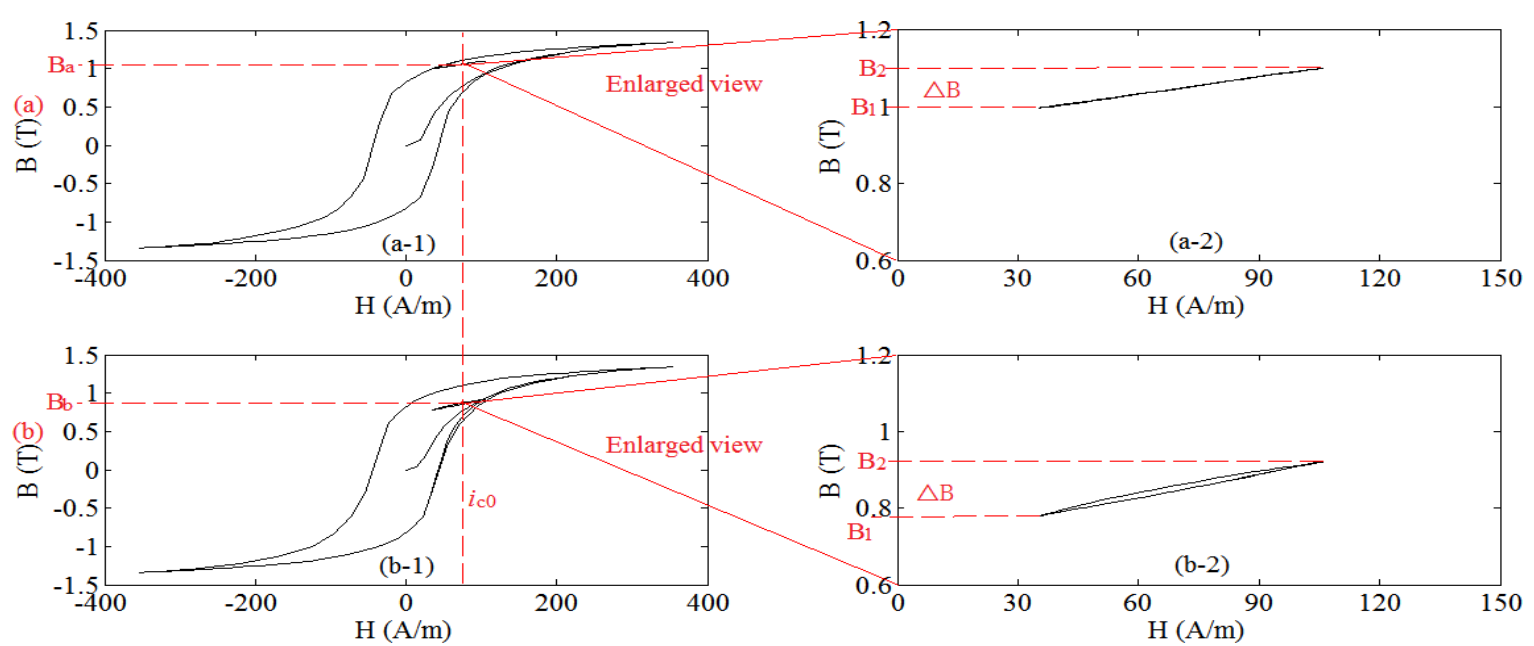

Fig. 14. Comparison on $B-H$ curves and enlargement of minor loops, Case 3; (a) down mode, namely higher magnetic flux density, and (b) up mode, namely lower magnetic flux density

Table 4. Iron losses and magnetic properties between the lower operating point $B_{\mathrm{b}}$ and the higher operating point $B_{\mathrm{a}}$, Case 3

\begin{tabular}{|c|c|c|}
\hline & $\begin{array}{c}\text { Up mode, } \\
\text { operating point } B_{\mathrm{b}}\end{array}$ & $\begin{array}{c}\text { Down mode, } \\
\text { operating point } B_{\mathrm{a}}\end{array}$ \\
\hline Iron loss $W_{\mathrm{Fe}}(\mathrm{W} / \mathrm{kg})$ & $3.1 \times 10^{-3}$ & $0.533 \times 10^{-3}$ \\
\hline $\begin{array}{c}\text { Classical eddy current loss } \\
W_{\text {eddy,classic }}(\mathrm{W} / \mathrm{kg})\end{array}$ & $0.531 \times 10^{-3}$ & $0.287 \times 10^{-3}$ \\
\hline Hysteresis loss $W_{\mathrm{hys}}(\mathrm{W} / \mathrm{kg})$ & $2.569 \times 10^{-3}$ & $0.246 \times 10^{-3}$ \\
\hline Magnetic flux density $B(\mathrm{~T})$ & 0.857 & 1.049 \\
\hline $\begin{array}{c}\text { Magnetic flux density } \\
\text { ripples } \Delta B(\mathrm{~T})\end{array}$ & 0.141 & 0.104 \\
\hline $\begin{array}{c}\text { Energy density of magnetic } \\
\text { field } \varepsilon\left(\mathrm{W} / \mathrm{m}^{3}\right)\end{array}$ & 31.814 & 37.347 \\
\hline
\end{tabular}

\section{Conclusion}

This paper presented numerical analysis and evaluation on the iron loss generated by minor loop of the reactor core used in a DC-DC converter with consideration of changes in the $\mathrm{ON}$ or OFF time of switching operation of the power semiconductor based on a magnetic hysteresis model using the play model. With the same average amplitude value $i_{\mathrm{c} 0}$ of the input current and its two different current waveforms which are the down and up modes shown in Figs. 9, 11, and 13 on the reactor core, the characteristics and sizes of the minor loops occurred at the two corresponding operating points of the magnetic flux density, i.e. $B_{\mathrm{a}}$ and $B_{\mathrm{b}}$ as described in Figs. 10, 12, and 14, respectively, are considerably different as expressed in Tables 2, 3, and 4, respectively.

Simulation results showed that the down-mode input current waveform corresponding to the higher magnetic flux density operating point $B_{\mathrm{a}}$ when we change the OFF time of the switching device can effectively reduce the iron loss caused by the minor loop because of the smaller size of the minor loop as well as have the smaller magnetic flux density ripples in the minor loop and the larger energy density of magnetic field with the minor loop as compared to the ones with the up-mode input current waveform corresponding to the lower magnetic flux density operating point $B_{\mathrm{b}}$ when we vary the $\mathrm{ON}$ time of the switching device. Moreover, the analysis results obtained in Case 2 and Case 3 have a similar trend with the results obtained in Case 1, namely, the higher magnetic flux density operating point $B_{\mathrm{a}}$ has the smaller iron loss, less magnetic flux density ripples and larger energy density of magnetic field than the ones at the lower magnetic flux density operating point $B_{\mathrm{b}}$. Obviously, the three simulation cases have confirmed the efficacy of the proposed calculation method.

In addition, since the input energy in the up and down modes is same and the iron loss caused by the minor loop in down mode is smaller than the one in up mode, the power conversion efficiency of the converter in down mode is higher than the one in up mode.

In next work, an experimental system will be developed to compare with the simulation results obtained in this study.

\section{Acknowledgment}

The authors would like to express sincere thanks to Dr. S. Satou for his enthusiastic support on simulation model.

\section{References}

( 1 ) S. Liu and K. Fujisaki: "Iron loss increase by power semiconductor characteristic of PWM inverter", IEE-Japan Industry Applications Society Conference, R1-4-2, Power Converter Application 1-67 (2012)

( 2 ) K. Fujisaki and S. Liu: "Magnetic hysteresis curve influenced by powersemiconductor characteristics in PWM inverter", Journal of Applied Physics, Vol.115, No.17, pp.17A321 (2014)

( 3 ) D. Kayamori and K. Fujisaki: "Influence of power semiconductor on-voltage on iron loss of inverter-fed", IEEE International Conference on Power Electronics and Drive Systems (2013)

(4) S. Odawara, D. Kayamori, and K. Fujisaki: "Iron loss characteristics of electrical steel sheet under inverter excitation by using power semiconductor with extremely low on-voltage property", Electrical Engineering in Japan, Vol.195, No.3, pp.58-66 (2016)

( 5 ) S. Odawara, K. Fujisaki, and F. Ikeda: "Proposal of numerical analysis technique for evaluating magnetic property in consideration of power semiconductor property in inverter using physical free energy model", IEEE Transactions on Magnetics, in press.

( 6 ) S. Bobbio, G. Miano, C. Serpico, and et al.: "Models of magnetic hysteresis based on play and stop hysterons", IEEE Transactions on Magnetics, Vol.33, No.6, pp.4417-4426 (1997)

( 7 ) T. Matsuo and M. Shimasaki: "An identification method of play model with input-dependent shape function", IEEE Transactions on Magnetics, Vol.41, No.10, pp.3112-3114 (2005)

( 8 ) S. Odawara, K. Fujisaki, T. Matsuo, and Y. Shindo: "Evaluation of magnetic 
properties considering semiconductor properties by using numerical technique coupling inverter circuit analysis to magnetic analysis", IEEJ Transactions on Industry Applications, Vol.135, pp.1191-1198 (2015)

( 9 ) N. Takahashi, S.H. Miyabara, and K. Fujiwara: "Problems in practical finite element analysis using Preisach hysteresis model", IEEE Transactions on Magnetics, Vol.35, No.3, pp.1243-1246 (1999)

(10) T. Matsuo: "Anisotropic vector hysteresis model using an isotropic vector play model", IEEE Transactions on Magnetics, Vol.46, No.8, pp.3041-3044 (2010)

(11) S.S. Zhong, S. Odawara, N.G.M. Thao, and et al.: "Numerical analysis on iron losses of reactor core under hysteresis $B-H$ curve using play model", IEEJ Meeting of Magnetics/Motor Drive/Linear Drive Joint Study Group, Dec. 13-14, 2018, Ritsumeikan University, Kyoto, Japan (2018)

(12) S. Odawara, S. Yamamoto, K. Sawatari, and et al.: "Iron loss evaluation of reactor core with air gaps by magnetic field analysis under high-frequency excitation", IEEE Transactions on Magnetics, Vol.51, No.11, pp.1-4 (2015)

Shuangshuang Zhong (Non-member) received the B.S. degree in

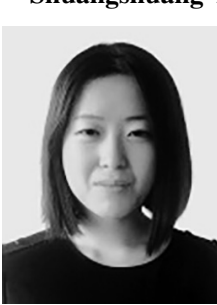
Logistics Engineering from Shenyang University of Technology, Shenyang, China, in 2009. She is a doctoral candidate in Mechanical Engineering from the Shenyang University of Technology, Shenyang, China. Now, she is as a special research student to study at Electromagnetic Energy System Laboratory, Toyota Technological Institute, Japan from March 2018. Her current research interests include reduction and suppression on vibration and noise of permanent magnet synchronous motor and iron losses of electrical motor.

Shunya Odawara (Member) received B. of Eng., M. Eng., and Dr.

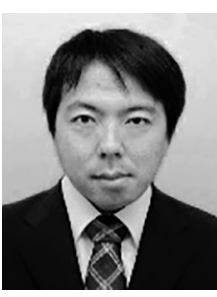
Eng. Degrees from Saga University, Saga, Japan, in 2009, 2010, and 2013, respectively. From 2013, he was in Toyota Technological Institute, Aichi, Japan as postdoctoral researcher. Now he is in Kitami Institute of Technology, Hokkaido, Japan as a research associate from 2017. His current research theme is loss characteristics evaluation by inverter excitation taking account of magnetic property and semiconductor property.

Nguyen Gia Minh Thao (Non-member) obtained B. Eng. (honors)

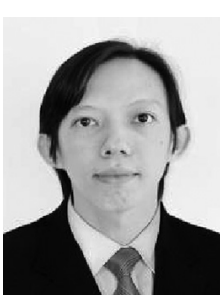
and M. Eng. (research) degrees in Electrical and Electronics Engineering from Ho Chi Minh City University of Technology (HCMUT), Vietnam, in March 2009 and April 2011, respectively. He obtained a Dr. Eng. degree in Electrical Engineering from Waseda University, Japan, in September 2015. In April 2009, he became a probationary Lecturer in the Faculty of Electrical and Electronics Engineering at HCMUT, where he has been a Lecturer. He was a Postdoctoral Research Associate at Waseda University, Japan from November 2015 to October 2017. He is currently a Postdoctoral Fellow at the Research Center for Smart Vehicles and Electromagnetic Energy System Laboratory, Toyota Technological Institute, Japan from November 2017. His research interests include intelligent control, advanced control methods, power electronics, electric motor drives, motor losses evaluation, renewable energy systems, and energy network optimization. He is a member of the Institute of Electrical and Electronics Engineers (IEEE) and Asian Control Association (ACA).
Keisuke Fujisaki (Senior Member) received the B. Eng., M. Eng.,

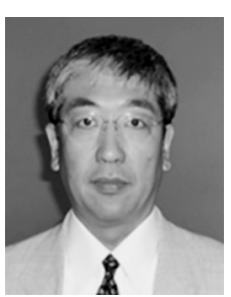
and Dr. Eng. degrees in Electronics Engineering from the Faculty of Engineering, The University of Tokyo, Tokyo, Japan, in 1981, 1983, and 1986, respectively. From 1986 to 1991, he conducted research on electromagnetic force applications to steel-making plants at the Ohita Works, Nippon Steel Corporation. From 1991 to 2010, he was a chief researcher in the Technical Development Bureau, Nippon Steel Corporation, Futtsu, Japan. Since 2010, he was a professor of Toyota Technological Institute. His current scientific interests are magnetic multi-scale, electromagnetic multi-physics, high efficient motor drive system, electrical motor, and power electronics. In 2002-2003, he was a Visiting Professor at Ohita University. In 2003-2009, he was a Visiting Professor at Tohoku University. Dr. Fujisaki received the Outstanding Prize Paper Award at the Metal Industry Committee sessions of the 2002 IEEE Industry Applications Society Annual Meeting.

Fujiyuki Iwamoto (Member) studied Oxide Electronics in The Uni-

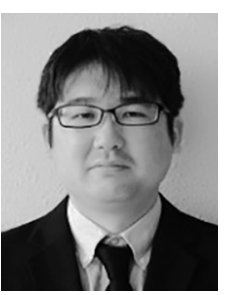
versity of Tokyo and in 2011. Since 2011, he has been with DENSO CORPORATION, Japan, where he is engaged in research and development of power electronics for automotive systems. From 2014 to 2016, he was engaged in designing ignition circuits for gasoline engines. Recently, his main research interest has been high-efficiency motor drives.

Tomonori Kimura (Member) received a M. degree in Crystalline

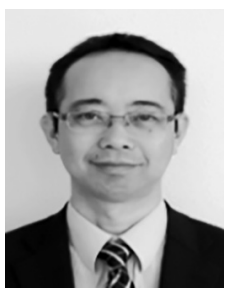
Materials Science from Nagoya University in 1994 Since 1994, he was engaged in the research and development of the power conversion technology for automotive in DENSO CORPORATION. Presently, he is working on the study of the high-efficiency motor drive system.

Takahiro Yamada (Member) received the M. degree in Informa-

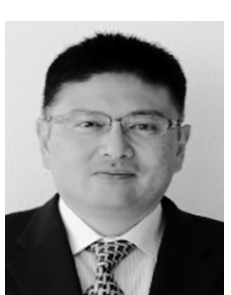
tion Engineering from Nagoya University, Nagoya, Japan in 1996. Since 1996, he has been with DENSO CORPORATION, Japan, where he is engaged in research and development of power electronics for automotive systems. Recently, his main research interest has been high-efficiency power conversion system and motor control. 